\title{
Sistema escolar chileno: características y consecuencias de un modelo orientado al mercado
}

\author{
Chilean school system: characteristics and consequences \\ of a market-oriented model
}

\author{
Cristóbal Villalobos / cristobal.villalobos@mail.udp.cl \\ Universidad Diego Portales, Chile \\ María Luísa Quaresma / quaresma.ml@gmail.com \\ Universidad Autónoma de Chile, Chile
}

\begin{abstract}
Considering the orientation towards competition and market that many educational systems have taken over last decades, this paper analyzes the characteristics, consequences and implications of the installation of these logics in the educational field from a paradigmatic case study: the Chilean school system. The article shows how this system is organized from the resignation of the role of the state in education, the centrality of private schools, the implementation of demand subsidies and the implementation of strong accountability, which has enhanced and deepened educational segregation, reducing the freedom of choice and generating social mobilization in the country, producing marked changes in the structures, processes and educational outcomes. Against this reality, we discuss the necessity and possibility of building a new educational logic, based on reflectivity, development of critical thinking, intelligent accountability and integral education.
\end{abstract}

Key words: competition-oriented system, marketization, privatization, accountability, Chilean school system.

Resumen: Al considerar la orientación hacia la competencia y el mercado que han adoptado múltiples sistemas educativos durante las últimas décadas, el artículo analiza las características, consecuencias y principales implicancias de la instalación de estas lógicas en el campo educacional, a partir del estudio de un caso paradigmático: el sistema escolar chileno. Así, se muestra cómo este sistema, organizado en torno a la dimisión del rol del Estado, la centralidad de lo privado, la implementación de subsidios a la demanda y la generación de fuertes políticas de rendición de cuentas, ha potenciado y profundizado fenómenos de segregación educativa, reducción de la libertad de elección y movilización social en el país, produciendo transformaciones en las estructuras, procesos y resultados educativos. Frente a este escenario, se discute la necesidad y posibilidad de construir una nueva lógica educacional, basadas en la reflexividad, el desarrollo del espíritu crítico, el accountability inteligente y la formación integral.

Palabras clave: orientación a la competencia, mercantilización, privatización, rendición de cuentas, sistema escolar chileno. 


\section{Introducción. Mercado y competencia en los sistemas educativos contemporáneos ${ }^{1}$}

Uno de los temas más discutidos en los recientes debates educativos de los países de la Organización para la Cooperación y el Desarrollo Económico (OCDE) es la permeabilidad de los sistemas escolares a las lógicas de la mercantilización. En las últimas décadas, múltiples países (por medio de formas heterogéneas y con distintos niveles de intensidad) han introducido en su agenda educativa conceptos importados de la esfera económica, como la competencia, la eficiencia, la eficacia, el accountability, la evaluación de desempeño y la privatización. Esta tendencia se ha acentuado desde los años setenta, momento en que los llamados Estados de Bienestar Social empiezan a tener dificultades para soportar los costos de las políticas sociales en periodos de recesiones económicas que, en los años recientes, se han agudizado aún más. En este escenario, diversas voces empiezan a cuestionar la centralidad del Estado en las distintas esferas de su accionar, postulando que éste viviría una profunda crisis de legitimidad y que no sería capaz de lograr su máxima eficacia, y defendiendo, por lo tanto, la sustitución de estas tareas por parte del sector privado (Estêvão, 1988).

En el campo educativo, como constata Van Zanten (2000), la reducción de los presupuestos destinados a la educación pública, la degradación de las condiciones de enseñanza resultante de la apertura de la escuela a niños sin el sistema de disposiciones escolares que poseen los "herederos" como los llamarían Bourdieu y Passeron (1964), la conjunción entre la inflación y desvalorización de los títulos académicos y las cada vez más constantes crisis económicas, han fortalecido la retórica liberal de los que defienden "(...) el modelo de mercado como la única solución posible para los males de la educación pública" (Van Zanten, 2000: 355).

Por otro lado, las recomendaciones de los organismos internacionales, como el Banco Mundial o el Banco Interamericano de Desarrollo, también tienen como eje central el potenciamiento de principios como la calidad, la eficiencia y la competencia, los cuales van siendo diseminados a través de los múltiples mecanismos de influencia que estas organizaciones tienen en las políticas educativas nacionales (Dale, 1999).

1 Los autores agradecen el apoyo del Centro de Estudios del Conflicto y la Cohesión Social (COES), financiado por la Comisión Nacional de Ciencia y Tecnología de Chile (CONICYT/FONDAP/15130009), y del CONICTY - FONDECYT Iniciación Numero 11140241. 
Así impera, en la actualidad y de manera global, una convergencia alrededor de un conjunto de tendencias que están en estrecha interconexión con el entendimiento de la educación como una mercancía y una concepción de los padres y estudiantes como consumidores, cuyas necesidades deberán ser satisfechas por los centros educativos que, en "un sistema de cuasi-mercado (...) son inducidos a ganar sus clientes a través de sus iniciativas, su trabajo en equipo, la calidad de su trabajo y de su oferta educativa" (Maroy, 2008: 2).

Entre estas tendencias, se destaca la promoción de la libre elección de las escuelas, que sería la expresión de la libertad individual, y la introducción del sistema de vouchers, que permitiría a los padres el ejercicio de esa libertad a través de la elección de la escuela que consideran de mejor calidad y más adaptada al perfil de su hijo (Maroy, 2007). Otros ejemplos de estas orientaciones son el potenciamiento de sistemas de evaluación externa de los alumnos, escuelas y sistemas educativos, que son concebidos como instrumentos de información capaz de proveer a las familias datos cuantitativos fácilmente comparables sobre los rendimientos de las escuelas (Mons, 2009); la promoción de la autonomía de los establecimientos, que es considerada como uno de los más importantes prerrequisitos para su eficacia (Chubb y Moe, 1990); y, finalmente, la profundización y desarrollo de procesos de privatización del sistema.

Esta privatización puede asumir, como plantean Ball y Youdell (2007), dos formas distintas: una más encubierta, la endógena; otra más visible, la exógena. En el primer tipo, las escuelas públicas importan estrategias y modus operandi del sector privado para adaptarse a un ideal de escuela-empresa y funcionar con la eficacia, eficiencia y sentido de competencia que caracteriza a las organizaciones empresariales (Ball, 2013a). En el segundo tipo, los servicios de educación pública se abren a la participación del sector privado, mediante el financiamiento público de escuelas privadas y/o la internalización de servicios escolares (transporte de alumnos, servicios de casino) a empresas privadas a quienes el Estado garantiza, por estas vías, la posibilidad de desarrollar actividades lucrativas (Ball, 2013a).

Simultáneamente y debido al énfasis en la calidad y a la presión para la racionalización y la eficacia de los sistemas, otro término también de la esfera empresarial pasa a ser utilizado en el campo educativo: el accountability. Éste suele traducirse, según Corvalán (2006), como "rendición de cuentas" y "responsabilización”, lo cual significa que el accountability "en parte es una rendición a los interesados o involucrados por los resultados del proceso educativo, lo que a su vez se espera tenga como consecuencia un aumento de los niveles de responsabilización de cada actor sobre tal proceso" (Corvalán, 2006: 11). 
En general, la aplicación de exámenes estandarizados de alto impacto y la construcción de rankings de las escuelas son dos de los más transnacionales mecanismos en los que se expresa la rendición de cuentas y la responsabilización por los resultados del aprendizaje. Un rasgo común a las distintas formas de accountability educacional es, sin embargo, la sanción (Konidari, 2012; Afonso, 2012), la cual se entiende como consecuencia directa de la evaluación de todos los involucrados en el proceso educativo: profesores, alumnos, establecimientos escolares y el sistema en general.

De hecho, la evaluación ha sido uno de los pilares centrales de este nuevo orden educativo, y es parte de lo que se denominó como el ascenso del "Estado Evaluador", un término introducido por primera vez por Neave (1988). Dicho término se buscó contraponer con el paradigma del "Estado Educador", característico de las sociedades de bienestar social y que fue el responsable de garantizar los servicios educativos, siendo reemplazado por un nuevo concepto: el "Estado Evaluador y Regulador".

Así, definir las grandes orientaciones y metas a alcanzar y establecer un sistema de control y evaluación para verificar si los objetivos han sido logrados (Lessard et al., 2002) son ahora los objetivos de este nuevo Estado. Como hemos dado cuenta, esta cultura de evaluación no se focaliza en los procesos pedagógicos sino en los resultados, lo cual ha provocado profundos cambios en los sistemas educativos y significativas reconfiguraciones en los ethos de las escuelas (Moreno, 2011).

En síntesis, la idea de que la escuela debe, al igual que una empresa, orientarse por la evaluación y competencia, es decir, por una lógica de gestión que responde también a las actuales demandas y preocupaciones de las familias, en especial de aquellas que pertenecen a las franjas medias y que ante la fragilidad de sus posiciones sociales y profesionales (antes garantizadas a través de la escolarización) se plantean como pilares centrales de los sistemas escolares, por sobre la importancia de la calidad de la enseñanza y la elección de una buena escuela.

Ahora bien, es importante mencionar que será la clase media la que, en los hechos, se verá beneficiada con muchas de las actuales políticas educacionales, las cuales, en general, sirven a sus intereses de clase (Ball, 2013a). Tal es, por ejemplo, el caso de la política de elección de escuela que asegura "enormes oportunidades para que la clase media utilice sus habilidades y recursos de manera que pueda aumentar o mantener sus privilegios en relación a las oportunidades educacionales y sociales" (Ball, 2013a: 464).

Ante este escenario internacional de reconfiguración del sistema edu- 
cativo, parece pertinente reflexionar sobre esta nueva retórica sobre la educación, el funcionamiento del sistema escolar y el rol del Estado, tomando como base la realidad educativa de uno de los países del mundo con un sistema educativo más mercantilizado y liberalizado: Chile. ¿Cómo se estructura el sistema escolar chileno? ¿Cuáles son los efectos de su permeabilidad a un sistema orientado al mercado? ¿Qué pueden aprender de la experiencia chilena otros países con sistemas educativos todavía protegidos de estas lógicas liberalizantes?

El presente artículo contestará a estas preguntas a través de una revisión profunda de las principales y más actuales investigaciones realizadas en Chile sobre este sistema. Se entregará una comprensión detallada y profunda de lo que ha pasado, a lo largo de estas últimas tres décadas, en: i) el sistema educativo chileno y sus consecuencias, como la (re)organización del sistema escolar (basada en el entendimiento de la educación como una mercancía y como un objeto de lucro), la dimisión del Estado de su rol de educador, el fortalecimiento y centralidad de lo privado y la generación de mecanismos de libre elección; y ii) en la (re)conceptualización del proceso educativo, que permite la profundización de procesos de segregación escolar y la generación de una concepción de educación circunscrita al aprendizaje de contenidos "escolarmente rentables" en los momentos de rendición de cuentas, o sea, en los momentos de evaluación.

\section{El sistema educativo chileno como caso único. Orientación al mercado y competencia}

Sin lugar a dudas, el sistema escolar chileno puede ser analizado como un caso único y particular en el concierto internacional. Existe consenso entre investigadores de la educación, cientistas sociales e historiadores de que las reformas llevadas adelante por la dictadura de Pinochet desde 1980 pueden entenderse como el momento fundacional de un nuevo modelo político, económico y social, que convirtió radicalmente la educación en el nivel primario, secundario y superior (Donoso, 2005; Mönckeberg, 2005; Oliva, 2010; Ruiz, 2013). Las principales transformaciones del sistema se desarrollaron a nivel institucional, normativo y financiero. Así, en pocos años se generó una transferencia y desconcentración de la administración de los establecimientos educacionales desde el Estado a los municipios, se cambió la forma de asignación de los recursos a las escuelas desde un pago de presupuestos hacia un pago de subvención por asistencia de alumnos (Sapelli y Aedo, 2001), se propició y fomentó el surgimiento de escuelas privadas con financiamiento 
estatal (Aedo, 2000) y se desmanteló y disminuyó el estatus laboral de los docentes (Ávalos, 2002; Oliva, 2008).

En general, la lógica y sentido de estos cambios apuntó a la construcción de un sistema basado en la competencia y organizado en torno a la idea de mercado educativo. Siguiendo las ideas del liberalismo económico y con una fuerte influencia de Milton Friedman, los encargados técnicos y políticos de la revolución tecnocrática inspirada en las ideas neoliberales (Pinto et al., 2006) generaron transformaciones del sistema que buscaron desarrollar un modelo donde oferentes y demandantes transaran libremente un bien que se consume (la educación), buscando así emular la lógica y funcionamiento de los mercados económicos en el espacio educativo.

Este experimento extremo fue implementado a partir de shocks sucesivos en la población, basados en la persecución, tortura y desaparición de opositores y en la generación de políticas radicales de ajuste social y económico (Klein, 2007). Asimismo, se generó un proceso de reconstrucción del rol del Estado en la sociedad y en el sistema educativo, el cual dejó de tener una función directiva e interventora y pasó a ocupar un rol subsidiario en el funcionamiento y organización de los servicios sociales en general, y de la educación en particular (Nef, 2007).

Posteriormente, con la generación de la transición pactada durante los años noventa (Garretón, 2000; Moulian, 1997), se inaugura un proceso de transformación sociopolítica que tiene como objetivo principal afrontar la globalización contemporánea y emprender un proceso de modernización de la sociedad, entendida como la búsqueda —-dentro de los marcos del sistema capitalista- de mayores niveles de crecimiento económico y equidad social (Garretón, 1994). En educación, este proceso se caracteriza por un aumento de la influencia del Estado, que incrementa notablemente los recursos públicos destinados a la educación (Peña y Brunner, 2007; Raczynski y Muñoz, 2007), la generación de modificaciones en las condiciones del proceso pedagógico y de los establecimientos educativos y el desarrollo de una serie de proyectos focalizados tendientes a apoyar académicamente a las escuelas de menores rendimientos (Programa P-900, Mece-Básica, Mece-Media, LEM, ECBI, Plan de Apoyo Compartido, entre otros). A pesar de estos cambios, la estructura y lógica de operación del sistema se mantienen prácticamente inalteradas hasta hoy en día (Donoso, 2005; Picazo, 2013). 


\section{Características del sistema escolar chileno: Centralidad de lo privado, institucionalidad de mercado, subsidio a la demanda, libertad de elección y accountability}

La construcción de un sistema escolar fundamentado en el mercado y la competencia se ha cristalizado en una serie de políticas y características del modelo educativo, que se han desarrollado con continuidades y rupturas desde los años ochenta. En general, pueden reconocerse cuatro principales características que definen el sistema: i) la construcción de un sistema mixto en términos de su propiedad, con un fuerte desarrollo del sector privado; ii) la consolidación de un sistema de financiamiento basado en el subsidio a la demanda; iii) la institucionalización del lucro y el copago como mecanismos de organización del sistema; y iv) la generación y desarrollo de importantes incentivos y castigos a escuelas, docentes y estudiantes.

Respecto al sistema mixto de propiedad, lo novedoso de este periodo no es tanto la existencia de distintos tipos de establecimientos (municipal o pública, particular subvencionada y particular pagada), sino la creciente importancia que va adquiriendo la educación privada, y la consecuente pérdida de matrícula y peso de la educación pública (Bellei et al., 2010).

Mientras en 1981, cerca del 15\% de la matrícula era privada, en 2010 este número se incrementaba por sobre el $40 \%$, con la consecuente disminución de la matrícula pública en más de $30 \%$ durante el periodo. Este proceso de privatización se ha visto desarrollado con base en la creación de miles de nuevas escuelas privadas, la mayoría de éstas no religiosas (Elacqua, 2012). Esto ha generado que actualmente Chile sea uno de los países con mayor participación privada en la educación escolar (OCDE, 2010). El crecimiento del sector privado ha generado un creciente proceso de segmentación socioeconómica de los establecimientos, ya que las escuelas públicas tienden a concentrar a los estudiantes vulnerables, mientras que las escuelas subvencionadas incluyen a estudiantes de nivel medio-bajo, medio y medio alto, y las escuelas privadas atienden a los estudiantes de la élite (García-Huidobro y Bellei, 2003).

Uno de los elementos que permitió y fomentó este proceso de privatización es la estructura de financiamiento del sistema (Aedo, 2000). Construido bajo la idea de que los establecimientos públicos y privados deberían tener el mismo trato por parte del Estado, se diseña un sistema de financiamiento basado en el subsidio a la demanda y donde el monto de dinero que recibía la escuela (municipal o particular subvencionada) varía de acuerdo al nivel de asistencia de los estudiantes durante el año. 
El objetivo de este mecanismo fue doble: por una parte, potenciar la libertad de elección de las familias, y por otro, aumentar la competencia entre las escuelas bajo el supuesto de que así éstas mejorarían progresivamente su calidad (Elacqua y Santos, 2013). El monto del financiamiento fue prácticamente igual para todo tipo de estudiantes hasta el año 2009, cuando se aprobó la Subvención Escolar Preferencial (SEP) que entrega recursos adicionales a los establecimientos que reciben estudiantes más vulnerables (Raczynski et al., 2013) y cuyos resultados preliminares parecen ser positivos - aunque pequeños y mixtos en sus magnitudes - en la calidad escolar (Mizala y Torche, 2013) pero sin efectos relevantes en la equidad (Valenzuela et al., 2013).

Una tercera característica del sistema es la instalación del copago y el lucro como aspectos organizacionales del sistema. Por una parte, el copago o financiamiento compartido se instaura desde 1993 y se entiende como un mecanismo a través del cual las escuelas pueden requerir que las familias aporten monetariamente a la educación de sus hijos, sin perder por ello el beneficio del subsidio estatal. Este beneficio es ocupado por dos tercios de los establecimientos particulares subvencionados, que pueden cobrar hasta $\$ 80.000$ mensuales —aproximadamente 40USS - (Simonsen, 2012). De manera complementaria, el sistema ha permitido la existencia de instituciones con fines de lucro; esto es, cuyos sostenedores puedan retirar los excedentes para fines personales (Bellei, 2013a), con el objetivo de permitir la proliferación y multiplicación de proyectos educativos diversos (Chumacero y Paredes, 2008), el fomento a la libertad de enseñanza y también la libertad de empresa y el potenciamiento del sistema de mercado.

Finalmente, el sistema escolar se ha construido en torno a fuertes mecanismos de rendición de cuentas, conocidos internacionalmente como procesos de accountability. La lógica general detrás del accountability es que las escuelas y sus actores se comportan de forma racional, por lo que establecer premios y sanciones permite orientar su actuar de mejora manera (Au, 2007). En Chile, desde los años ochenta se ha producido una profundización del sistema de rendición de cuentas, principalmente por dos vías. Por un lado, a través de la construcción de una estructura de evaluación de los establecimientos educativos, consolidando toda una institucionalidad de control y análisis de establecimientos (Agencia de Calidad, Superintendencia de Cali$\mathrm{dad}$, entre otros). Junto con esto, se ha promovido y fomentado la utilización de las pruebas estandarizadas - especialmente a través del Sistema de Medición de la Calidad Escolar, SIMCE - como mecanismo de control y comparación de las escuelas, los cuales se han intensificado durante los últimos años (Cassasus, 2010; Waissbluth, 2013). 


\section{Principales consecuencias del sistema. Segregación educativa, libertad limitada de los padres y procesos de movilización social}

La aplicación del sistema de orientación al mercado y la competencia por más de 30 años ha generado una serie de consecuencias en términos de la calidad, equidad, eficiencia y eficacia del sistema. Si bien la investigación ha sido bastante fructífera durante la última década, aún no es posible dimensionar la totalidad de los efectos en el corto, mediano y largo plazo que produciría la aplicación de este modelo. Aun así, en términos generales, es posible reconocer tres grandes resultados de este proceso.

En primer lugar, estudios generados principalmente en los últimos años han revelado la existencia de altos niveles de segregación entre establecimientos (Bellei, 2013b; Hernando et al., 2014), los cuales en términos generales se han elevado durante el último periodo (Elacqua, 2012; Valenzuela et al., 2014; Villalobos y Valenzuela, 2012). En esta línea, se ha comprobado que, en términos socioeconómicos, la segregación entre escuelas se produce desde los primeros años de escolaridad, en magnitudes elevadas y estables a través de los distintos años del ciclo escolar. Por otra parte, se ha indicado que la segregación académica entre escuelas es especialmente elevada en la enseñanza media (Valenzuela et al., 2014; Villalobos y Valenzuela, 2012).

Adicionalmente, la evidencia ha demostrado que la segregación socioeconómica es mucho más elevada en los colegios particulares subvencionados que en los municipales, al menos durante los primeros años de escolaridad (Elacqua, 2012; Mizala y Torche, 2012; Valenzuela et al., 2010). Asimismo, comparaciones internacionales de la segregación han indicado que Chile posee los niveles más altos de la OCDE, ocupando los dos primeros lugares en las mediciones de 2006, 2009 y 2012 (OCDE, 2010; Valenzuela et al., 2010, 2014).

En general, los análisis de los factores que incidirían en estos niveles de segregación muestran el efecto de distintos elementos, entre los cuales destacan las preferencias de los padres, que buscan un tipo de escuela específico para sus hijos donde éstos compartan con otros niños similares (Wormald et al., 2012), el diseño institucional del sistema, especialmente a través del financiamiento compartido (Mizala y Torche, 2012), y los niveles de segregación residencial, que refuerzan dinámicas segregadoras que el sistema escolar potencia y amplifica (Elacqua y Santos, 2013; Valenzuela et al., 2014).

Finalmente, estudios recientes han mostrado la existencia de relevantes niveles de segregación al interior de las escuelas, los cuales se producen 
principalmente a través de criterios académicos y en los establecimientos más vulnerables (Treviño et al., 2014), profundizando así la segregación entre establecimientos.

En segundo término, distintos estudios se han focalizado en mostrar las limitaciones al proceso de elección de padres y estudiantes. Por una parte, diversas investigaciones se han especializado en estudiar el comportamiento de los padres, revelando que el supuesto de elección por calidad en general no se cumple y dando cuenta del impacto de factores culturales, socioeconómicos y geográficos en la elección escolar (Canals, 2013; Elacqua y Fábrega, 2004; Flores y Carrasco, 2013; Gallego y Hernando, 2009; Raczynski y Hernández, 2011), lo cual limitaría fuertemente la idea de competencia entre instituciones.

Por otra parte, un conjunto de estudios han analizado las posibilidades reales de elección de los padres, dando cuenta de que éstas son limitadas, ya sea por el financiamiento compartido (Elacqua et al., 2012; Mizala y Torche, 2012) o por la generación de procesos de selección (abiertos o velados) de estudiantes. En esta línea, recientes investigaciones sobre la materia han revelado que las prácticas selectivas se encuentran instaladas e institucionalizadas en el sistema (Carrasco et al., 2014; Contreras et al., 2010; Godoy et al., 2014), siendo transversales al tipo de dependencia del establecimiento.

A modo de ejemplo, García-Huidobro (2007) estima, utilizando la encuesta complementaria para padres del SIMCE, que el proceso de selección se da en un $73 \%$ de los casos. En general, se reconoce que requisitos como la aplicación de entrevistas personales a los padres o la observación de sesiones de juego del niño (Madero y Madero, 2012), además de requisitos como mostrar certificados de matrimonio, bautizo, de renta, son altamente utilizados por los establecimientos (Carrasco et al., 2014; Godoy et al., 2014).

En términos generales, se reconoce que la lógica detrás de la aplicación de estos mecanismos de selección en un sistema de mercado es la generación de eficiencias a partir del descreme o selección de estudiantes que posean habilidades o características socioeconómicas actuales o potenciales más altas (Hsieh y Urquiola, 2003; Mizala y Torche, 2012). Asimismo, se ha dejado en evidencia que la aplicación de mecanismos de accountability estaría generando procesos de estrechamiento de los tipos de proyectos educativos en el sistema, los que se enfocarían de manera relevante en los aspectos académicos de la formación por sobre otros aspectos (Villalobos y Salazar, 2014). En último término, no se han podido demostrar efectos positivos de la existencia de establecimientos con fines de lucro en la calidad escolar ni en la eficiencia 
del sistema, lo que ha generado cuestionamientos respecto de la institucionalidad educativa vigente (Bellei, 2013a; Contreras et al., 2011; Huneeus, 2011; Villalobos, 2014).

Finalmente, y como consecuencia - en algunos casos directa, en otros indirecta - de los efectos anteriormente destacados, se ha producido un creciente proceso de movilización social y descontento con el modelo educativo. Así, y utilizando como consigna la transformación del modelo como horizonte de cambio (Mayol, 2012), se han producido crecientes procesos de movilización social de estudiantes, padres y docentes. En general, se reconoce que este proceso de movilización comienza a desplegarse con fuerza a comienzos de la década del 2000, y adquiere puntos de inflexión en las manifestaciones estudiantiles y sociales de los años 2006 y 2011, en las que se combinaron elementos de movilización social clásicos con diseños organizativos, estéticos y socioculturales novedosos (Bellei et al., 2014; Donoso, 2013; Salinas y Fraser, 2012).

Lo fundamental de estos procesos de movilización es que han potenciado una discusión política y ética respecto de la transformación de la lógica de la competencia y la orientación al mercado del sistema educativo chileno. Con variaciones, esta discusión se ha desarrollado en el ámbito comunicacional y de los medios de comunicación de masas (Cabalín, 2014), en la investigación educativa (Bellei et al., 2010; García-Huidobro y Falabella, 2013; Varios Autores, 2012) y en la ciudadanía y la discusión pública (Fleet, 2011; Orellana, 2012), generando amplios espacios de discusión respecto del sentido, lógica y características del sistema escolar chileno.

\section{Conclusiones. ¿Hacia nuevos sistemas educativos? Lecciones y aprendizajes a partir del caso chileno}

La permeabilidad del sistema educativo chileno a las lógicas de la mercantilización es visible, como esta reflexión ha mostrado, en tres niveles: i) en las estructuras educativas; ii) en los procesos educativos; y iii) en los resultados educativos. En cuanto al primer nivel, la adhesión chilena al mercado educativo tuvo impactos en la organización de la red escolar, a través del potenciamiento y centralidad que se le dio al sector privado, y en la dimisión del Estado de la administración educativa.

A nivel de los procesos, es claro que se han producido cambios no solamente en la esfera de las escuelas - desde un punto de vista organizacional (selección de alumnos y segregación escolar) y de las prácticas pedagógica 
(acción docente direccionada hacia el entrenamiento para obtención de una buena evaluación) - sino también en la esfera familiar (papel activo de las familias en la elección de la escuela) y en la esfera individual (pérdida del valor intrínseco del aprendizaje, que es reemplazado por la lógica de aprender para lograr los resultados).

Finalmente, este sistema parece haber producido cambios en la conceptualización de lo que es la educación y de sus resultados; desde una concepción de un bien común hacia una basada en una mercancía de consumo, lo cual también ha generado una transformación de los fines de la educación, pasando de un concepto de educación entendido como promoción de la reflexividad y desarrollo del espíritu crítico, a una idea de educación como eficacia y eficiencia en la resolución de tests estandarizados.

Como plantea Ball, esto conlleva el desarrollo de un proceso de internalización de una nueva lógica, ya que “(...) el neoliberalismo no es algo que existe solamente allá, afuera, en la economía, [sino también] es algo que también existe acá, adentro, en nuestras cabezas" (Ball, 2013a: 462). Esto implica que las transformaciones en las estructuras y procesos educativos han generado (y se han visto potenciadas) por la introyección de nuevos ideales y valores que la educación orientada al mercado ha desarrollado, transformando a su vez el proceso mismo y el producto de la educación y, además, los sentidos que le atribuyen los actores, o sea, los significados que los sujetos dan a los actos de educar y de aprender.

Así, y como también lo ha planteado Stobart (2010), la generación de un sistema escolar con un énfasis en el sistema de evaluación y de rendición de cuentas ha empobrecido el hecho mismo de educar, ya que los alumnos van perdiendo el interés intrínseco por el aprendizaje y se preocupan solamente con los resultados de los exámenes y/o en la obtención de un título académico.

Según el autor, esto implica que ahora "el objetivo es obtener un resultado determinado en vez de enriquecer el aprendizaje” (Stobart, 2010: 149). Por ello, dicho sistema de evaluación no produciría una mejora sostenible en la educación y en la sociedad en general, contribuyendo, en cambio, para el refuerzo de la desigualdad, segmentación y segregación social, consecuencias que, como evidenciamos, también han acompañado la introducción de los distintos mecanismos de reforma educativa con orientación al mercado en Chile.

Ahora bien, ¿cómo salir de esta crisis profunda a la que ha llegado la educación? Una de las propuestas a tener en cuenta para el modelo vigente en Chile y de los demás países que insisten en la lógica de la competencia y el 
mercado es la desarrollada por Stephen Ball, quien nos habla de la necesidad de un "back to basics" (Ball, 2013b: 4); es decir, de una reconceptualización del modelo educativo que tenga como centro un retorno a lo básico, que implique repensar cuáles deberán ser los objetivos de la educación, cuál debería ser el significado de ser educado, cuál debería ser el rol de la escuela y —lo más importante- quiénes deberían decidir estos asuntos.

De esta forma, se pone el acento en que las transformaciones no debieran ser decididas por los "autoproclamados expertos" (Ball, 2013b: 25) ni por los representantes de los intereses económicos que han dictado las actuales políticas educativas, sino por el conjunto de actores escolares, incluyendo a padres, alumnos, profesores y directivos.

En esta misma línea, el sistema escolar chileno y demás países también debieran generar una reconceptualización respecto de uno de los ejes centrales de este modelo educativo orientado al mercado: el accountability. Una propuesta en este sentido es la desarrollada por Stobart (2010), quien propone la instalación de otro modelo de accountability: el inteligente. Éste es definido como una forma de rendición de cuentas y de promoción de la responsabilidad basada más en los valores y en la confianza en los profesores y las escuelas y menos en las sanciones. Bajo esta mirada, los mecanismos de evaluación deberían ser múltiples y más sofisticados, y las metas propuestas para los alumnos más realistas, tomando en consideración las condiciones de cada estudiante: sus tiempos, constreñimientos, recursos materiales y capacidades humanas.

De manera similar, Howe y Aschraft (2005) proponen la construcción de una evaluación más democrática y deliberativa basada en el principio de la inclusión, el diálogo y de la deliberación, es decir, en la generación de una evaluación caracterizada por una participación activa de los involucrados en el proceso, o, dicho de otra forma, en una relación dialógica en la que todos los sujetos expresen sus intereses y puntos de vista y, a través de la cooperación y concesiones mutuas, puedan llegar a conclusiones.

Finalmente, es claro que los sistemas educativos mercantilizados, en especial el chileno, necesitan un rediseño de sus lógicas de financiamiento, organización y funcionamiento interno, que potencien la eficiencia, la eficacia y la inclusión, y donde el Estado juegue un rol garante en la entrega de los procesos educativos entendidos como un derecho social, donde no existan procesos de selección discriminadores y donde no hayan incentivos en contra del propio proceso educativo, tal como han reconocido organismos internacionales como la propia OCDE y el Fondo de las Naciones Unidas para la Infancia (Unicef). 
Estas propuestas de transformación estructural tienen en común la generación de una nueva mirada sobre la educación, que no se base en la instrucción como foco y que vaya más allá de la mera transmisión, memorización y rendimiento académico (Ballion, 1991). Asimismo, esta educación plena y multidimensional tiene en su base una (nueva) concepción de excelencia, ahora escrita en plural, como ha defendido Bourdieu ya desde la década de 1980. Esta excelencia, que el autor denomina de largo alcance, evitaría la generación de sentencias sin apelación (Bourdieu, 1987: 108) para los estudiantes que no encajan en la monolítica definición de éxito.

Desde esta perspectiva, y en línea con los más actuales planteamientos de Hutmacher (1995), el proceso de educar se entendería como el desarrollo de un conjunto de disposiciones y aptitudes en el alumnado, entre las que destacan el saber aprender, saber analizar, comprender y reflexionar, ser capaz de comunicar, argumentar y ser creativo. Pero significaría, aún más importante, que la escuela sería capaz de dotar los alumnos de las disposiciones que les permitan construir un proyecto profesional pleno de sentido (Postic, 1995) y constituirse como "agentes de un proyecto de vida moralmente autónomo" (Bárcena et al., 1999: 38).

De esta forma, invertir la tendencia de la mercadización y mecanización de la educación exige, de parte de las escuelas y de las políticas públicas al menos cuatro acciones: $i$ ) una mayor preocupación con el desarrollo de las competencias morales, sociales y emocionales de los jóvenes (Good y Weinstein, 1995); ii) la promoción de experiencias de índole deportiva y cultural que promuevan la mejora de la autoestima y del autoconcepto de los estudiantes y que faciliten la generación de redes de relaciones sociales (West y Hopkins, 1996); iii) el desarrollo de una apuesta en la dimensión cultural de la educación, o sea, en la formación de "hombres y mujeres de cultura" que han adquirido los saberes socialmente reconocidos como dignos de interés intelectual o estético (Ballion 1991) y que manejen los instrumentos intelectuales que le permiten atribuir sentido a estos hechos; y iv) la promoción de la ciudadanía, formando ciudadanos libres, críticos, que intervienen en la vida cívica y que asumen responsabilidades sociales (Perrenoud, 2005).

Finalmente, es importante considerar la siempre tensa relación entre sociedad y sistema educativo. Dado que la escuela no es un mundo aparte de la sociedad, este nuevo modelo de educación, que podría constituir una alternativa viable, es siempre de difícil implementación en contextos políticosociales marcados por lógicas donde la competencia y la mercadización están validadas cultural y socialmente. Como dice Perrenoud (2005), la educación, por sí sola no puede hacer milagros mientras no se produzcan cambios en la 
estructura sociocultural y económica de la sociedad que apunten a reemplazar la competencia por la solidaridad y la formación por la educación.

Pese a ello, los movimientos estudiantiles, escuelas críticas y otras acciones sociales, como el Mayo de 68, la pedagogía crítica de Paulo Freire o el Movimiento Chileno de los Pingüinos, tienen también un rol en la producción de estos cambios, como nos han demostrado las luchas de estudiantes, profesores y padres alrededor del mundo. En definitiva, es en el re-pensar de la educación como concepto y como praxis y en el re-conceptualizar los principios de las políticas públicas que queda la esperanza para el cambio en el (des)orden mundial en que vivimos. Reflexionar sobre el rumbo de estas políticas - como intenta hacer este artículo - es la tarea central para investigadores, docentes, padres y estudiantes, pues permitirá abrir pistas y profundizar los debates sobre posibles alternativas a este modelo.

\section{Bibliografía}

Aedo, Cristian (2000), La educación privada en Chile: Un estudio histórico-analitico desde el periodo colonial hasta 1990, Santiago, Chile: RIL Editores.

Afonso, Almerindo (2012), "Para uma concetualização alternativa de accountability em educação", en Educação \& Sociedade, vol. 33, núm. 119, Portugal: CEDES.

Au, Wayne (2007), "High-Stakes Testing and Curricular Control: A Qualitative Metasynthesis", en Educational Research, año 36, Inglaterra: Routledge Journals, Taylor \& Francis Ltd.

Ávalos, Beatrice (2002), Profesores para Chile. Historia de un proyecto, Santiago, Chile: Ministerio de Educación.

Ball, Stephen (2013a), "Entrevista com Stephen J. Ball - Privatizações da educação e novas subjetividades: contornos e desdobramentos das políticas (pós) neoliberais", en Revista Brasileira de Educação, vol. 18, núm. 53, Brasil: Associação Brasileira de Pesquisadores em Educação Especial - ABPEE.

Ball, Stephen (2013b), Education, justice and democracy: The struggle over ignorance and opportunity, Inglaterra: Center for Labour and Social Studies.

Ball, Stephen y Deborah Youdell (2007), "La privatisation déguisée dans le secteur éducatif public", Rapport préliminaire presentado en el Internationale de l'éducation -5ème congrès mondial.

Ballion, Robert (1991), La bonne école, París: Hatier.

Bárcena, Fernando et al. (1999), “La dimensión ética de la educación”, en Bárcena, Fernando et al., La escuela de la ciudadanía. Educación, ética y politica, Bilbao: Desclée de Brouwer.

Bourdieu, Pierre (1987), "Propostas para o ensino do futuro", en Cadernos de Ciências Sociais, núm 5, Portugal: Edições Afrontamento.

Bourdieu, Pierre y Jean-Claude Passeron (1964), Les héritiers, París: Les Editions de Minuit. 
Convergencia Revista de Ciencias Sociales, núm. 69, 2015, Universidad Autónoma del Estado de México

Bellei, Cristian (2013a), “El 'fin de lucro' como política educacional”, en García-Huidobro, Juan Eduardo y Alejandra Falabella [eds.], Los fines de la educación, Santiago, Chile: Ediciones UC.

Bellei, Cristian (2013b), "El Estudio de la segregación socioeconómica y académica de la educación Chilena”, en Estudios pedagógicos, año 39, Chile: Facultad de Filosofía y Humanidades Universidad Austral de Chile.

Bellei, Cristian, Cristian Cabalín y Víctor Orellana (2014), “The 2011 Chilean student movement against neoliberal educational policies", en Studies in Higher Education, año 39, Inglaterra: Routledge Journals, Taylor \& Francis Ltd.

Bellei, Cristian, Daniel Contreras y Juan Pablo Valenzuela (2010), Ecos de la Revolución Pingüina. Avances, debates y silencios de la reforma educacional, Santiago, Chile: Pehuén.

Bellei, Cristian, Pablo González y Juan Pablo Valenzuela (2010), "Fortalecer la Educación Pública: Un desafío de interés nacional”, en Bellei, Cristian, Pablo González y Juan Pablo Valenzuela [eds.], Ecos de la Revolución Pingüina. Avances, desafíos y silencios de la reforma educacional, Santiago, Chile: Pehuén.

Cabalín, Cristian (2014), “The conservative response to the 2011 chilean student movement: Neoliberal education and media”, en Discourse: Studies in the Cultural Politics of Education, Inglaterra: Routledge Journals, Taylor \& Francis Ltd.

Canals, Catalina (2013), Factores sociales y geográficos que inciden en la elección de escuela, Chile: Universidad de Chile.

Carrasco, Alejandro, Francisca Bogolasky, Carolina Flores, Gabriel Gutiérrez y Ernesto San Martín (2014), "Selección de estudiantes y desigualdad educacional en Chile: ¿Qué tan coactiva es la regulación que la prohibe?", Informe resumen proyecto FONIDE 711286, Chile: CEPPE, Pontificia Universidad Católica de Chile.

Cassasus, Juan (2010), "Las reformas basadas en estándares. Un camino equivocado”, en Bellei, Cristian, Daniel Contreras y Juan Pablo Valenzuela [eds.], Ecos de la Revolución Pingüina. Avances, debates y silencios en la Reforma Educativa, Santiago, Chile: Pehuén.

Chubb, John y Terry Moe (1990), Politics, Markets and America's Schools, Washington: Brookings Institution Press.

Chumacero, Romulo y Ricardo Paredes (2008), “Should for-Profit School Be Banned?”, en MPRA, Paper núm. 15099, Alemania: University Library of Munich.

Contreras, Dante et al. (2011), "El lucro en educación escolar. Evidencia y desafíos regulatorios", en Trabajos de Investigación en Politicas Públicas, núm. 10, Chile: Departamento de Economía, Universidad de Chile.

Contreras, Dante, Paulina Sepúlveda y Soledad Bustos (2010), "When schools are the ones that choose: The effects of screening in Chile", en Social Science Quarterly, año 91, Estados Unidos de América: Southwestern Social Science Association.

Corvalán, Javier (2006), “Accountability educacional: Rendición de cuentas más responsabilización por los procesos y resultados de la educación”, en Corvalán, Javier y Robert Mcmeekin, Accountability educacional: posibilidades y desafíos para América Latina a partir de la experiencia internacional, Santiago: San Marino. 
Cristóbal Villalobos y Maria Luísa Quaresma. Sistema escolar chileno: características y consecuencias de un modelo orientado al mercado

Dale, Roger (1999), "Specifying globalization effects on national policy: a focus on mechanisms”, en Journal of Educational Policy, vol. 14, núm. 1, Inglaterra: Routledge Journals, Taylor \& Francis Ltd.

Donoso, Sebastián (2005), "Reforma y política educacional en Chile: 1990-2004. El neoliberalismo en crisis", en Estudios Pedagógicos, año 31, Chile: Facultad de Filosofía y Humanidades Universidad Austral de Chile.

Donoso, Sofía (2013), "Dynamics of change in Chile: Explaining the emergence of the 2006 pingüino movement”, en Journal of Latin American Studies, año 45, Inglaterra: Institute of of Latin American Studies.

Elacqua, Gregory (2012), “The impact of school choice and public policy on segregation: Evidence from Chile", en International Journal of Educational Development, año 32, núm. 3, Orlando: Elsevier Educational Research Program.

Elacqua, Gregory y Rodrigo Fábrega (2004), El consumidor en la educación. El actor olvidado de la libre elección de escuelas en Chile, Chile, Santiago: Programa de Promoción de la Reforma Educativa en América Latina y el Caribe (PREAL).

Elacqua, Gregory y Humberto Santos (2013), "Los efectos de la elección escolar en la segregación socioeconómica en Chile: Un análisis georreferenciado”, en Documento de Referencia, núm. 1, Santiago, Chile: Espacio Público.

Estêvão, Carlos (1998), "A construção da autonomia e a autonomia da gestão nas escolas privadas”, en Revista Portuguesa de Educação, vol. II, núm. 1, Portugal: Universidade do Minho.

Fleet, Nicolás (2011), "Movimiento estudiantil y transformaciones sociales en Chile: Una Perspectiva Sociológica”, en Revista Polis, año 10, Santiago, Chile: Universidad de Los Lagos, Campus Santiago.

Flores, Carolina y Alejandro Carrasco (2013), "Preferencias de los padres, libertad de elección y Segregación”, en Documento de Referencia, Santiago, Chile: Espacio Público.

Gallego, Francisco y Andrés Hernando (2009), "On the determinants and implications of School choice: Structural estimates and Simulations for Chile”, Documento de Trabajo, IE-PUC núm. 343, Santiago, Chile: Pontificia Universidad Católica de Chile.

García-Huidobro, Juan Eduardo (2007), "La selección de alumnos en la Ley General de Educación”, en Política educativa. Revista Docencia, núm. 32, Santiago, Chile: Colegio de Profesores de Chile.

García-Huidobro, Juan Eduardo y Cristian Bellei (2003), "Desigualdad educativa en Chile”, en Hevia, Renato [ed.], La Educación en Chile Hoy, Santiago, Chile: Universidad Diego Portales.

García-Huidobro, Juan Eduardo y Alejandra Falabella (2013), Los fines de la educación, Santiago, Chile: Ediciones UC.

Garretón, Manuel Antonio (1994), La Faz Sumergida del Iceberg: Estudios sobre la transformación cultural, Santiago, Chile: CESOC, LOM Ediciones.

Garretón, Manuel Antonio (2000), La sociedad en que vivi(re)mos. Introducción sociológica al cambio de siglo, Santiago, Chile: LOM. 
Godoy, Felipe, Felipe Salazar y Ernesto Treviño (2014), "Prácticas de selección en el sistema escolar chileno: Tipos de requisitos de postulación, legitimación en el sistema y las debilidades de la Ley", Informes para la Politica Educativa, Santiago, Chile: Centro de Políticas Comparadas de Educación, Universidad Diego Portales.

Good, Thomas y Rhona Weinstein (1995), "As escolas marcam a diferença: evidências, críticas e novas perspetivas", en Nóvoa, António, As organizaçôes escolares em análise, Lisboa: Publicações Dom Quixote.

Hernando, Andrés, Karina Niklitschek y Daniel Brieba (2014), Segregación escolar en Chile, Santiago: Horizontal.

Howe, Kenneth y Catherine Ashcraft (2005), "Deliberative democratic evaluation: successes and limitations of an evaluation of school choice", en Teachers College Records, vol. 107, núm. 10, Estados Unidos de América: Columbia University.

Hsieh, Chang-Tai y Miguel Urquiola (2003), "When school compete, how do they compete? An Assessment of Chile's nationwide school voucher program”, en NBER Working Paper, Cambridge, MA: National Bureau of Economic Research.

Hutmacher, Walo (1995), "A escola em todos os seus estados: das políticas de sistemas às estratégias de estabelecimento", en Nóvoa, António, As organizaçóes escolares em análise, Lisboa: Publicações Dom Quixote.

Huneeus, Federico (2011), “¿De qué se trata finalmente el debate del lucro?”, en Revista Docencia, año 45, Chile: Colegio de Profesores.

Klein, Noemi (2007), La doctrina del shock. El auge del capitalismo de desastre, Madrid, España: Paidós.

Konidari, Victoria (2012), "Privatização da educação pública - necessidade incontestável ou fim de um mito?”, en Educação Em Foco, núm. 20, Brasil: Faculdade de Educação da Universidade Federal de Juiz de Fora.

Lessard, Claude, André Brassard y Jacques Lusignan (2002), Les tendances des politiques éducatives en matière de structures et de régulation, d'imputabilité et de reddition de comptes: les cas du Canada (Ontario et Colombie-Britannique), de la France et du Royaume-Uni, Montréal: LABRIPROF-CRIFPE, Faculté des Sciences de l'Education, Université de Montréal

Madero, Cristóbal y Ignacio Madero (2012), “Elección escolar y selección estudiantil en el sistema escolar chileno. ¿Quién elige a quién? El caso de la educación católica”, en Revista Mexicana de Investigación Educativa, año 17, México: Consejo Mexicano de Investigación Educativa.

Mayol, Alberto (2012), El derrumbe del modelo. La crisis de la economia de mercado en el Chile contemporáneo, Santiago, Chile: LOM.

Maroy, Christian (2007), "Les modes de régulation del'École: Une comparaison européenne”, en Revue Internationale d'Éducation, núm. 46, Francia: Centre International d'Études pédagogiques.

Maroy, Christian (2008), "Por qué y cómo regular el mercado educativo?", en Profesorado. Revista de currículum y formación del profesorado, vol. 12, núm. 2, España: Universidad de Granada. 
Cristóbal Villalobos y Maria Luísa Quaresma. Sistema escolar chileno: características y consecuencias de un modelo orientado al mercado

Mizala, Alejandra y Florencia Torche (2012), "Bringing the schools back in: The stratification of educational achievement in the Chilean voucher system", en International Journal of Educational Development, año 32, núm. 1, Orlando: Elsevier Educational Research Program.

Mizala, Alejandra y Florencia Torche (2013), “¿Logra la Subvención Escolar Preferencial igualar los resultados educativos?”, en Documento de Referencia, núm. 9, Santiago, Chile: Espacio Público.

Mons, Nathalie (2009), "Effets théoriques et réels des politiques d'évaluation standardisée", en Revue Française de Pédagogie, núm. 169, Francia: École Normale Supérieure de Lyon.

Mönckeberg, María Olivia (2005), La Privatización de las Universidades. Una historia de dinero, poder e influencias, Santiago, Chile: Copa Rota.

Moreno, Tiburcio (2011), "La cultura de la evaluación y la mejora de la escuela”, en Perfiles Educativos, vol. XXXIII, núm. 131, México: UNAM.

Moulian, Tomás (1997), Anatomía de un mito, Santiago, Chile: LOM.

Neave, Guy (1988), "On the Cultivation of Quality, Efficiency and. Enterprise: an overview of recent trends in higher education in Western Europe, 1986-1988”, en European Journal of Education, vol. 23, núm. 1/2, Estados Unidos de América: Wiley-Blackwell.

Nef, Jorge (2007), "El concepto de Estado subsidiario y la educación como un bien de mercado: Un bosquejo de análisis político", en Enfoques Educacionales, año 2, Chile: Facultad de Ciencias Sociales de la Universidad de Chile.

ocde (2010), Pisa 2009 Results: What Students Know and Can Do - Student Performance in Reading, Mathematics and Science, vol. 1, OCDE.

Oliva, María Angélica (2010), "Política educativa chilena 1965-2009. ¿Qué oculta esa trama?”, en Revista Brasileira de Educação, año 15, Brasilia: ANPED.

Oliva, María Angélica (2008), "Políticas educativas y la profundización de la desigualdad en Chile”, en Estudios Pedagógicos, año 34, Chile: Facultad de Filosofía y Humanidades Universidad Austral de Chile.

Orellana, Víctor (2012), "Sobre el malestar social con la educación y la energía del movimiento social. El primer paso del Chile del siglo XXI”, en Varios Autores, Es la Educación, Estúpido!, Santiago, Chile: Ariel.

Peña, Carlos y José Joaquín Brunner (2007), La reforma al Sistema Escolar. Aportes al debate, Santiago, Chile: Universidad Diego Portales.

Perrenoud, Philippe (2005), Escola e cidadania. O papel da escola na formação para a democracia, Porto Alegre: Artmed Editora.

Picazo, María Inés (2013), Las politicas escolares de la concertación durante la transición democrática, Santiago, Chile: Universidad Diego Portales.

Pinto, Julio, Rolando Álvarez y Verónica Valdivia (2006), Su revolución contra nuestra revolución. Izquierdas y derechas en el Chile de Pinochet (1973-1981), vol. 1, Santiago, Chile: LOM.

Postic, Marcel (1995), Para uma estratégia pedagógica do sucesso escolar, Porto: Porto Editora. 
Convergencia Revista de Ciencias Sociales, núm. 69, 2015, Universidad Autónoma del Estado de México

Raczynski, Dagmar y Macarena Hernández (2011), Elección de colegio: imágenes, valoraciones y conductas de las familias y segregación social escolar, Santiago, Chile: Asesorías para el Desarrollo.

Raczynski, Dagmar y Gonzalo Muñoz (2007), "Reforma educacional chilena: El difícil equilibrio entre la macropolítica y la micropolítica”, Documento de Trabajo, Santiago, Chile: CIEPLAN.

Raczynski, Dagmar et al. (2013), "Subvención Escolar Preferencial (SEP) en Chile: Un intento por equilibrar la macro y micropolítica escolar", en Revista Iberoamericana sobre Calidad, Eficacia y Cambio en Educación, año 11, España: Red Iberoamericana de Investigación sobre Cambio y Eficacia Escolar (RINACE).

Ruiz, Carlos (2013), “Educación y democracia: Aportes para el cambio desde la demanda estudiantil”, en García-Huidobro, Juan Eduardo y Alejandra Falabella [eds.], Los fines de la educación, Santiago, Chile: Ediciones UC.

Salinas, Daniel y Pablo Fraser (2012), "Educational opportunity and contentious politics: The 2011 chilean student movement", en Berkeley Review of Education, año 3, Estados Unidos de América: Graduate School of Education at the University of California, Berkeley.

Sapelli, Claudio y Cristian Aedo (2001), "El sistema de vouchers en educación: Una revisión de la teoría y evidencia empírica para Chile”, en Estudios Públicos, año 82, Santiago, Chile: CEP.

Simonsen, Elizabeth (2012), Mala Educación: Historia de la revolución escolar, Santiago, Chile: Debate.

Stobart, Gordon (2010), Tiempos de pruebas: los usos y abusos de la evaluación, Madrid: Morata.

Treviño, Ernesto, Juan Pablo Valenzuela y Cristóbal Villalobos (2014), Segregación académica y socioeconómica al interior de la escuela. Análisis de su magnitud, evolución y principales factores explicativos, Santiago: CPCE-CIAE.

Valenzuela, Juan Pablo, Cristian Bellei y Danae De Los Ríos (2010), “Segregación escolar en Chile”, en Martinic, Sergio y Gregory Elacqua [eds.], ¿Fin de ciclo? Cambios en la Gobernabilidad del sistema educativo, Santiago, Chile: OREALC, Unesco, Pontificia Universidad Católica de Chile.

Valenzuela, Juan Pablo, Cristian Bellei y Danae De Los Ríos (2014), “Socioeconomic school segregation in a market-oriented educational system. The case of Chile", en Journal of Education Policy, año 29, Inglaterra: Routledge Journals, Taylor \& Francis Ltd.

Valenzuela, Juan Pablo, Gabriel Villaroel y Cristóbal Villalobos (2013), "Ley de Subvención Escolar Preferencial (SEP): Algunos resultados preliminares de su implementación”, en Pensamiento Educativo. Revista de Investigación Educacional Latinoamericana, año 50, Santiago, Chile: Pontificia Universidad Católica de Chile.

Van Zanten, Agnès (2000), "Un libéralisme éducatif sans frontières?”, en Van Zanten, Agnès, L'école l'état des savoirs, París: Éditions La Découverte.

Varios Autores (2012), Es la Educación, Estúpido!, Santiago, Chile: Ariel. 
Cristóbal Villalobos y Maria Luísa Quaresma. Sistema escolar chileno: características y consecuencias de un modelo orientado al mercado

Villalobos, Cristóbal (2014), "El lucro en el sistema escolar chileno. Elementos empíricos y conceptuales para entender la necesidad de su prohibición", en Revista Docencia, Chile: Colegio de Profesores.

Villalobos, Cristóbal y Felipe Salazar (2014), "Proyectos educativos en el sistema escolar chileno: Una aproximación a las libertades de enseñanza y elección”, Informe para la Politica Educativa, núm. 2, Santiago, Chile: Centro de Políticas Comparadas en Educación.

Villalobos, Cristóbal y Juan Pablo Valenzuela (2012), "Polarización y cohesión social del sistema escolar chileno”, en Revista de Análisis Económico, año 27, Estados Unidos de América: ILADES, Georgetown University.

Waissbluth, Mario (2013), Cambio de Rumbo. Una nueva via chilena a la educación, Santiago, Chile: Debate.

West, Mel y David Hopkins (1996), "Reconceptualising school effectiveness and school improvement", Trabajo presentado en el American Educational Research Association Annual Meeting "School improvement and school effectiveness: towards a new synthesis".

Wormald, Guillermo et al. (2012), "Cultura de cohesión e integración en las ciudades chilenas”, en EURE, año 76, Santiago, Chile: Pontificia Universidad Católica de Chile.

Cristóbal Alejandro Villalobos Dintrans. Magíster en Economía Aplicada, Universidad Alberto Hurtado-Georgetown University (Chile-EUA). Labora en el Centro de Políticas Comparadas de Educación, Universidad Diego Portales, Chile. Líneas de investigación: sociología de la educación, economía de la educación, calidad y equidad educativa, segregación escolar, educación superior, sociología del conocimiento. Publicaciones recientes: Villalobos, Cristóbal, "El lucro en el sistema escolar chileno. Elementos empíricos y conceptuales para entender la necesidad de su prohibición”, en Revista Docencia, núm. 53, Chile: Colegio de Profesores (2014); Brunner, J. J. y Villalobos, C., Politicas de Educación Superior en Iberoamérica, 2009-2013. Un análisis comparado, Santiago: Ediciones UDP (2014); Valenzuela, J. P., Villaroel, G. y Villalobos, C., "Ley de Subvención Escolar Preferencial (SEP). Algunos resultados preliminares de su implementación", en Pensamiento Educativo. Revista de Investigación Educacional Latinoamericana, vol. 50, núm. (2013).

Maria Luísa da Rocha Vasconcelos Quaresma. Doctorado en Sociología por la Universidade do Porto (Portugal). Labora en la Universidad Autónoma de Chile, Instituto de Estudios Sociales y Humanísticos, Facultad de Ciencias Sociales y Humanidades (profesora e investigadora) e Instituto de Sociología, Faculdade de Letras da Universidade do Porto (investigadora asociada). Líneas de investigación: excelencia académica, educación de las élites, mercantilización de la educación. Publicaciones recientes: Quaresma, 
Maria Luísa y Abrantes, Pedro, "Schooling the elites, schooling the poors. Contrasting socialization contexts within the Portuguese educational system", en Lap Lambert Academic Publishing (2014); Quaresma, Maria Luísa, "A excelência no plural em colégios singulares", en Torres, Leonor, Entre mais e melhor escola, Lisboa: Editora Mundos Sociais (2014); Quaresma, Maria Luísa, "Práticas culturais dos jovens das classes sociais dominantes: reflexões em torno do ecletismo cultural”, en Fórum Sociológico, núm. 23 (2013).

Recepción: 2 de octubre de 2014.

Aprobación: 6 de mayo de 2015. 